\title{
First memorial issue in honor of Dr. Meng-Sing Liou
}

\author{
C.-H. Chang ${ }^{1}$ (I) $\cdot$ H. Luo $^{2}$ (I)
}

Published online: 17 June 2019

๑) Springer-Verlag GmbH Germany, part of Springer Nature 2019

It is our honor and distinct privilege to introduce this twovolume memorial tribute to our much loved and respected friend and colleague, Dr. Meng-Sing Liou. Meng-Sing was well known for enthusiasm for his research, dedication to his work, as well as his generosity and patience toward his colleagues and students. His untimely passing in 2017 has left a big vacuum in several diverse technical communities that his work impacted and on the personal level for those who had the fortune to be among his friends.

Dr. Liou is best known for his works on the development of the family of AUSM schemes, although his research spanned a variety of subjects in computational fluid dynamics, grid generation, turbulence modeling, combustion, multidisciplinary design, analysis and optimization, multiphase flows, and nonlinear flow modeling. Meng-Sing was an avid contributor to many research societies. He was a Fellow of the American Institute of Aeronautics and Astronautics (AIAA) and the International Shock Wave Institute (ISWI). He was also an editor of the Shock Waves journal since 2007 and a member of the Scientific Committee of the International Conference for Computational Fluid Dynamics (ICCFD).

In early 2018, two proposals to publish special memorial issues in honor of Dr. Liou were put forward by the Acta Mechanica Sinica and Shock Waves journals. Upon suggestion and guidance of Wei Shyy, we later decided to join these efforts and publish all papers in Shock Waves. In consultation with many people from Meng-Sing's immediate technical family, we came up with a list of contributors as we assessed their involvement in pursuing the frontiers defined by MengSing's work, both independently and in collaborations with him. We received 30 papers from his former students, postdocs, colleagues, and friends, who would like to pay tribute

C.-H. Chang

chchang@theofanous.net

H. Luo

hluo2@ncsu.edu

1 Theofanous Co. Inc., 93 Castilian Dr., Goleta, CA 93117, USA

2 Department of Mechanical and Aerospace Engineering, North Carolina State University, Raleigh, NC 27695, USA to his legacy in a series of articles inspired by his vision and his example. All submitted papers were subjected to the same rigorous peer review procedure as any regular contribution to Shock Waves. Each paper was evaluated by at least two or more international experts. Out of 30 submissions, 19 papers were accepted for publication. (Thirteen of them were accepted after one revision, five papers-after two revisions, and one paper-after three revisions.) Six papers were rejected after peer review, and the remaining five papers are still at various stages of processing.

In view of the large number of submissions, we organized these papers into two memorial issues. The present issue, Part I consisting of 10 papers, collects all manuscripts related to AUSM (Meng-Sing's crowning achievement), while Part II will include papers on design optimization, combustion, turbulence, free interface flows, as well as CFD works not directly related to AUSM. Part II will consist of the remaining nine already accepted papers and the five papers which are still under review (if accepted).

The story of the AUSM schemes can be traced back to the mid-1980s, when Meng-Sing started his cooperation with van Leer on the inviscid flux vector splitting (FVS) methods. One main drive at that time was to find an upwind scheme which would be as simple as the flux vector splitting method but would avoid excessive diffusion at the contact surface. The first breakthrough came in Meng-Sing and Steffen's 1992 paper [1]. In that paper, they wrote the convection fluxes as a product of a cell interface velocity (based on van Leer's Mach splitting function) and the upwind fluid states, while the pressure term was split in the same manner as in the FVS method. This makes the convection fluxes to be upstreambiased and, therefore, enables the new AUSM scheme to accurately capture the contact surface without excessive dissipation. The test results also showed that as far as shock wave capturing is concerned, AUSM is capable of performing as well as the much more sophisticated Godunov and Roe schemes.

In Meng-Sing's 1996 paper published in JCP [2], he proposed two new splitting functions for Mach function and pressure flux which satisfy a series of conditions for 

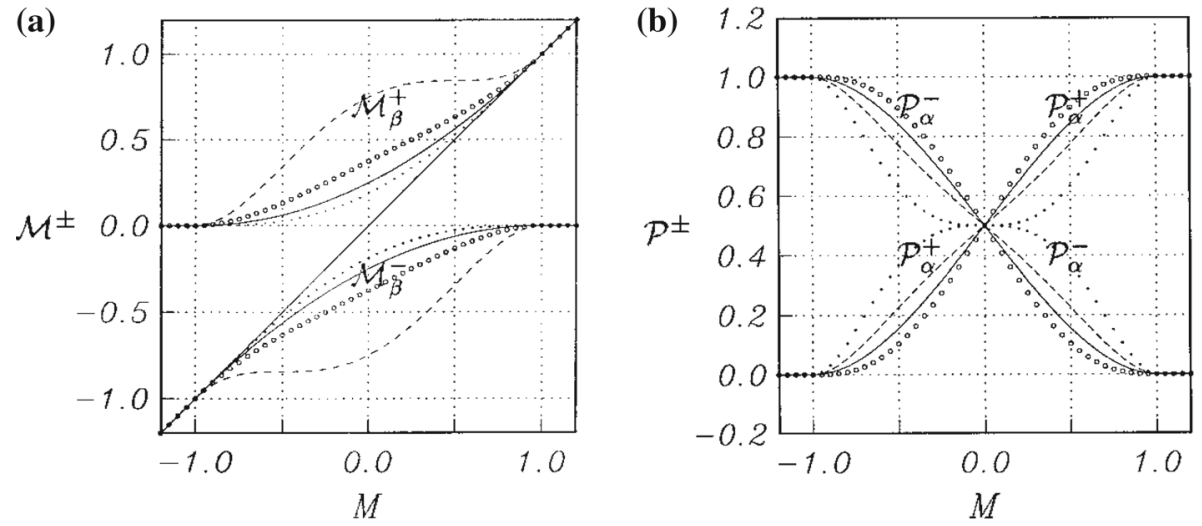

Fig. 1 Splitting functions for the $\operatorname{AUSM}^{+}$scheme: a Mach splitting function $\mathcal{M}^{ \pm}(M)$; b pressure splitting function $\mathcal{P}^{ \pm}(M)$. Reproduced from [2] with permission

consistency, monotonicity, symmetry, and continuous differentiability. These two splitting functions, as we show in Fig. 1, guarantee the smooth and monotonic transition of fluxes between two states. They are vital to the success of all the successive AUSM-family schemes and have not been altered thereafter.

In the same paper, Meng-Sing also showed that by using Prandtl's relation for the critical sound speed, it is possible to derive an interface sound speed which allows the exact resolution of the stationary shock. He clearly demonstrated that the new $\mathrm{AUSM}^{+}$scheme is capable of capturing stationary shock waves and contact discontinuities. In addition, $\mathrm{AUSM}^{+}$shows excellent properties in terms of resistance to shock instabilities, such as in the slow-moving shock problem, the carbuncle phenomenon for high Mach flows, and the even-odd grid perturbation issues for a moving shock. Compared to the Godunov and Roe schemes, the $\mathrm{AUSM}^{+}$method is also less prone to generation of a nonphysical discontinuity (the expansion shock) in a strong expansion flow.

Due to its simple form, the building of $\mathrm{AUSM}^{+}$schemes does not require full details of the eigensystem. This makes $\mathrm{AUSM}^{+}$highly suitable to be extended to various problems by modifying its numerical dissipation. Following MengSing's 1996 paper [2], a number of AUSM-type methods were proposed to improve its accuracy and robustness. It is to be especially mentioned that, due to their simplicity, the AUSM schemes were increasingly used in solving compressible multiphase flows $[3,4]$. The progress during that decade was later summarized in Meng-Sing's 2006 paper [5] on the $\mathrm{AUSM}^{+}$-up method ("u" and "p" represent the velocity- and pressure-based numerical dissipation terms). More details regarding this period may be found in the account of the early development of AUSM schemes presented in the current issue by Jack Edwards [6].
Having been initiated in the mid-1980s, Meng-Sing's efforts to pursue an ideal upwind scheme have spanned over 30 years. His last paper on the AUSM scheme was presented at the AIAA CFD conference in 2017 [7]. In that paper, he focused on anomalous overheating in the receding flow problem - an issue that had been mentioned by several authors before but was scarcely investigated. He carefully demonstrated that the cause of the overheating is that the discretization of conservation equations (mass, momentum, and energy) is not consistent with the continuous equation for entropy. This led him to propose an entropy equation to replace the energy equation in the $\mathrm{AUSM}^{+}$-up scheme to eliminate the overheating. In Fig. 2, we reproduce two figures from his 2017 paper [7] as a fitting concluding remark to epitomize his life's legacy.

We thank Bram van Leer for contributing the In Memoriam article for the present issue. Taken together with Jack Edwards's retrospective article, they provide a rather complete account of the advances in CFD owed to Meng-Sing. The other papers selected for this issue cover various topics ranging from the all-speed solver, MHD flows, and shock boundary simulations in a turbomachinery application to the carbuncle problem and compressible multiphase flows. We hope this issue presents a clear view of current developments of AUSM schemes and the exciting applications they enable.

Finally, we would like to thank all the authors who kindly contributed their papers to this memorial issue and were willing to revise them to meet our standards. The expertise and dedicated work of all reviewers were also crucial for ensuring the high quality of manuscripts appearing in this issue. We would like to express our sincere appreciation to Evgeny Timofeev and Andrew Higgins from Shock Waves for their tireless effort, hard work, and kind patience. Without their strong support and commitment, this memorial issue would not have been possible. 


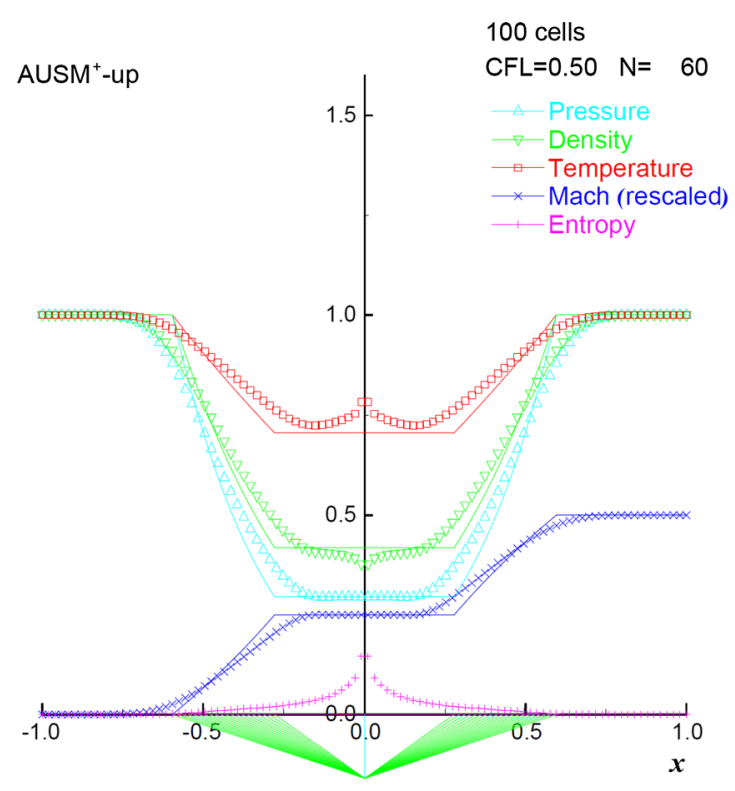

(a) $\mathrm{AUSM}^{+}$-up

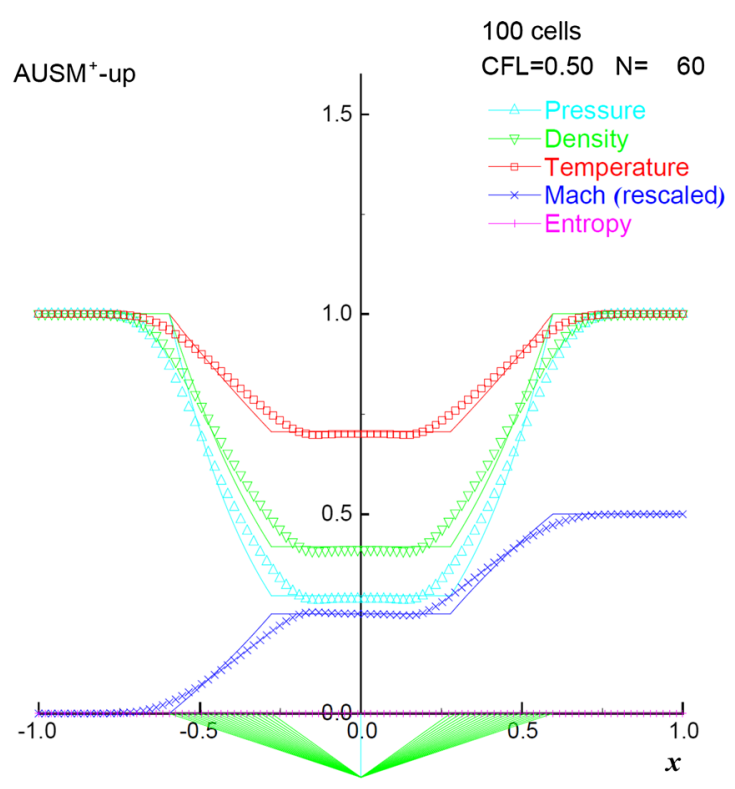

(b) $\mathrm{AUSM}^{+}$-up with s-equation

Fig. 2 Effects of enforcing the entropy equation: $\mathbf{a} \mathrm{AUSM}^{+}$-up and $\mathbf{b} \mathrm{AUSM}^{+}$-up with the entropy equation for the 1D receding flow problem $(M=0.8)$. Reproduced from [7] with permission

\section{References}

1. Liou, M.-S., Steffen, C.: A new flux splitting scheme. J. Comput. Phys. 107, 23-39 (1993). https://doi.org/10.1006/jcph.1993.1122

2. Liou, M.-S.: A sequel to AUSM: AUSM ${ }^{+}$. J. Comput. Phys. 129, 364-382 (1996). https://doi.org/10.1006/jcph.1996.0256

3. Edwards, J.R., Liou, M.-S.: Low-diffusion flux-splitting methods for flows at all speeds. AIAA J. 36(9), 1610-1617 (1998). https:// doi.org/10.2514/2.587

4. Chang, C.-H., Liou, M.-S.: A robust and accurate approach to computing compressible multiphase flow: Stratified flow model and AUSM $^{+}$-up scheme. J. Comput. Phys. 225, 840-873 (2007). https:// doi.org/10.1016/j.jcp.2007.01.007
5. Liou, M.-S.: A sequel to AUSM, part II: $\mathrm{AUSM}^{+}$-up for all speeds. J. Comput. Phys. 214(1), 137-170 (2006). https://doi.org/10.1016/ j.jcp.2005.09.020

6. Edwards, J.R.: Reflections on the early development of the "AUSM family" of Riemann solvers. Shock Waves (2019). https://doi.org/ 10.1007/s00193-018-0863-8

7. Liou, M.-S.: The root cause of the overheating problem. 23rd AIAA Computational Fluid Dynamics Conference, Denver, CO, AIAA Paper 2017-4107 (2017). https://doi.org/10.2514/6.2017-4107

Publisher's Note Springer Nature remains neutral with regard to jurisdictional claims in published maps and institutional affiliations. 